\title{
Pericardial diseases in the era of imaging, biomarkers and molecular diagnosis
}

\author{
Bernhard Maisch
}

Published online: 17 March 2013

(C) Springer Science+Business Media New York 2013

Pericardial diseases only affect a small proportion of patients with heart disease. But their diagnosis and differential diagnosis is, though too often neglected by cardiologists and internists, extremely important for the individual patient and of therapeutic and prognostic relevance in the differential diagnosis of cardiac symptoms [1, 2]: An excellent example for precordial pain is acute pericarditis, which should be differentiated from aortic dissection, myocardial infarction, pneumonia or pleuritis, pulmonary embolism, pneumothorax, costochondritis (Da Costa syndrome), gastroesophageal reflux or neoplasm and herpes zoster. In the spectrum of heart failure syndromes, it is pericardial constriction that causes dyspnoea and peripheral edema. Seferovic et al. particularly address these issues in their contribution, which was designed to update the 2004 guidelines of the European Society of Cardiology [3]. These guidelines are still the only guidelines worldwide on the management of pericardial diseases. Interventional pericardiologists nowadays use intrapericardial endoscopy and biopsy together with classic cytology to establish an etiologically based diagnosis $[4,5]$ by making use of techniques that have been accepted and applied to diagnose inflammatory cardiomyopathies [6] or tumors or rheumatic diseases since several decades. Pericardial access has become a vital interest for electrophysiologists, who now also ablate epicardial foci and reentry sites to treat malignant ventricular tachycardia [7, 8]. Pericardial access is

Bernhard Maisch: Former Director of the University Hospital of Internal Medicine and Cardiology.

\section{B. Maisch ( $\square)$}

Professor Emeritus, Faculty of Medicine, Philipps-University and UKGM GmbH Marburg, Feldbergstr. 45, 35033 Marburg, Germany

e-mail: bermaisch@t-online.de; bermaisch@aol.com also discussed for localized pharmacological treatment [9], even for stem cells or cocktails with growth factors [10]. Devices for locomotion on the epicardial surface have been designed and applied in preclinical settings [10, 11].

Apart from the patient's symptoms, echocardiography remains the mainstay of imaging of acute pericardial syndromes but also of constrictive pericarditis, effusive-constrictive pericarditis, pericardial effusion, tamponade, absence of the pericardium and cysts or tumors. The remarkable progress, which has been made in echocardiography in the last years, is very well described by Veress, Feng and Oh from the Mayo-Clinic. Progress in cardiac tissue Doppler analysis, strain and strain rate imaging by speckle tracking imaging and three-dimensional echocardiography, the assessment of early diastolic annulus velocity and annulus reversus by TDI improves the differentiation of constriction from restrictive myocardial disease and is in the focus of their contribution. Threedimensional echocardiography may come up as a useful method for the precise assessment pericardial masses as it provides incremental value to $2 \mathrm{D}$ echocardiography by detecting anatomical and pathological structures with higher accuracy. Of particular interest are their contributions and that of others on effusive-constrictive pericarditis [12-14] and on the differential diagnosis of constrictive pericarditis to restrictive cardiomyopathy [15].

Syed and coworkers in this issue point out that effusiveconstrictive pericarditis is best characterized in patients with tamponade who continue to have elevated intracardiac pressure after removal of pericardial fluid. The underlying causes are pericardial inflammation in conjunction with the presence of pericardial fluid under pressure, whereby the etiology is diverse.

Alter et al. add valuable information on imaging by reviewing MRI and CT [16, 17] as well as scintigraphic 
methods in the diagnosis of pericardial diseases: Fast spinecho T1-weighted sequences with black blood preparation permit morphological evaluations best. Fast spin-echo T2-weighted sequences with fat saturation and short tau inversion recovery sequences visualize edema and inflammation. Delayed inversion recovery imaging at 5-20 min subsequent to contrast agent administration demonstrate late gadolinium enhancement indicative for fibrosis or inflammation. Ventricular volumes and myocardial mass can be measured by steady-state free precession sequences, which are required to assess cardiac function, ventricular wall stress and impaired function in constrictive pericarditis, which in turn results from chronic inflammatory processes. They may lead to increased stiffness, which impedes the slippage of the pericardial and epicardial layers and thereby cardiac filling. CT assesses pericardial calcification best. PET provides unique information on the in vivo metabolism of 18 -fluorodeoxyglucose that can be superimposed on CT findings and is useful for identifying inflammatory processes or masses, for example, neoplasms.

In cardiac tamponade, in larger and smaller effusions, a safe pericardial puncture is of prime importance. Ristic and coworkers investigated the feasibility of the pericardial halo phenomenon, which delineates the epicardial fat pat. It has been rediscovered as highly sensitive radiologic feature for the detection of a pericardial effusion in chest X-ray already 60 years ago [18] and received a revival to orientate the puncturing needle to the pericardial sac particularly in the lateral view. They nicely demonstrated that the halo sign correlates well with the volume of the pericardial effusion estimated before pericardiocentesis by echocardiography and assessed thereafter directly. It can now be referred to as a guide to a safe pericardial puncture even in textbooks [19].

Pericardiocentesis is the immediate life-saving measure from tamponade. But it does not treat the cause of it. Entering the pericardium and sampling pericardial [20] and epicardial tissue under the control of a fiberglass endoscope, the pericardioscope, will permit further investigations. Pericardioscopy allows safe biopsies under optical control and has opened a new window to the heart [21, 22] as illustrated by convincing images and the contribution by Maisch and coworkers in the issue. An etiologically based intrapericardial therapy in malignant effusions $[23,24]$ or with triamcinolone in autoimmune perimyocardial disorders [25] depends on the yield and the diagnosis from targeted biopsy sampling. Pericardioscopy has opened a new diagnostic and therapeutic window to the heart.

An etiological diagnosis can be based on histology and molecular biology: Polymerase chain reaction on cardiotropic agents has been used for 2 decades for assessment from endomyocardial biopsies $[5,26]$ and was now reported for the first time in a truly large cohort of 259 consecutive patients with pericardial effusions by Pankuweit et al. Of note, autoreactive, virus-negative effusions were most frequently seen among patients with large effusions or tamponade in $35 \%$ of cases, when compared to infectious effusions in $14 \%$ and malignant effusions in $28 \%$ of the Marburg referral center over a period of 20 years.

Karatolios et al. report the discriminative signatures of biochemical markers in malignant and non-malignant pericardial effusion. Malignant pericardial effusions had significantly higher pericardial fluid levels of the tumor markers CEA, CA 19-9, CA 72-4, SCC and NSE, when compared to autoreactive effusions. Elevated pericardial CA 72-4 levels were associated with a high diagnostic accuracy for a malignant pericardial effusion as confirmed later by cytology and biopsy. Interestingly, also the discriminative properties of cytokeratin fragment 19, a tumor marker used in the evaluation of patients with lung cancer, could be of particular value in malignant effusions, since a high ratio of pericardial to serum osteoprotegerin and high levels of proapoptotic TNF-related apoptosis-inducing ligand (TRAIL) in the effusion were the best variable combination to predict malignancy [27].

Cytokine patterns in pericardial diseases are not well established. Ristic et al. have analyzed in patients with pericardial effusion proinflammatory interleukin (IL)-6 and tumor necrosis factor (TNF)-alpha as well as immunoregulatory cytokines such as transforming growth factor (TGF)-beta1 and interferon (IFN)-gamma and compared them with patients suffering from myocarditis and ischemic heart disease. They could identify a characteristic "high TNF-alpha/low TGF-beta1" cytokine serum pattern in viral pericarditis and a low IL-6 serum concentration in autoreactive pericardial effusions. A high IL-6 pericardial to serum ratio was the hallmark of autoreactive pericardial effusions, as was a high TNF-alpha and IFN-gamma pericardial to serum ratio in virus-positive pericardial effusions.

In etiologically, undefined pericardial effusion without exclusion of viral or bacterial origin oral cortisone should be given with caution. NSAIDs and Aspirin up to $1 \mathrm{~g}$ are still the mainstay of medical therapy. Imazio and Adler recommend in their contribution and based on the COPE trial the addition of colchicine in acute pericarditis [28]. Colchicine, as given in the CORE [29] and CORP trial [30], has been proposed the best available medical therapy in recurrent pericarditis. Since recurrent and chronic symptomatic pericarditis are the evidence of therapeutical efficacy, my intention is that in those patients an etiological diagnosis should be attempted and these forms should not be labeled idiopathic any longer. In cases with effusions of viral origin, treatment with corticoids might increase the 
recurrence rate, although they could be effectively treating inflammation at the beginning!

When the pericardial effusion is large and pericardiocentesis and tissue sampling has excluded the viral genome, intrapericardial triamcinolone is the treatment of choice [3, 4, 25]. Similarly, intrapericardial cis-platin or thiotepa together with systemic tumor treatment should be given to patients with malignant pericardial effusions [4, 23, 24], which conforms with the European guidelines [3].

Intrapericardial treatment with ethanol after evacuation of the "spring water fluid" of a pericardial cyst is also effectively preventing recurrence of this rare condition as outlined by 2 cases reported in this issue by Maisch.

Tuberculous pericarditis is rare in Europe but the most frequent form of pericardial effusion in Africa and other regions of the world where Mycobacterium tuberculosis is endemic. It has a caseload of approximately 80,000160,000 per year [31]. Ntsekhe and Mayosi in their contribution could show that HIV alters the natural history and outcomes of tuberculous pericarditis. In immunocompromised patients, constrictive pericarditis develops less frequently, but the patients have a higher mortality compared to immunocompetent pericarditis patients [32].

In an excellent overview, Cho and Schaff outline indications and limitations for cardiac surgery in pericardial diseases. Apart from the correction of anomalies like herniations, aortic dissection with pericardial effusion or localized symptomatic effusions, which cannot be reached by a puncturing needle, trauma, rare cases of highly painful acute pericarditis and, above all, constrictive pericarditis are the areas of surgical treatment. For constrictive pericarditis, the authors clearly recommend radical pericardiectomy.

All contributors and the guest editor sincerely hope that this unique issue of HFR dedicated to pericardial syndromes gives a stimulating up-to-date appraisal of our current knowledge in the diagnosis and therapy of pericardial diseases.

\section{References}

1. Shabetai R (2003) The pericardium. Kluwer, Boston

2. Spodick DH (1997) The pericardium: a comprehensive textbook. New York, Marcel Dekker

3. Maisch B, Seferović PM, Ristić AD et al (2004) Task force on the diagnosis and management of pericardial diseases of the European society of cardiology. European Society of cardiology guidelines: diagnosis and management of the pericardial diseases. Executive summary. Eur Heart 25(7):587-610

4. Maisch B, Ristić AD, Seferović M, Tsang SMT (2011) Interventional pericardiology-pericardiocentesis, pericardioscopy, pericardial biopsy, balloon pericardiotomy, and intrapericardial therapy. Springer, Heidelberg
5. Maisch B, Pankuweit S (2012) Current treatment options in (peri)myocarditis and inflammatory cardiomyopathy. Herz 37:644-656

6. Maisch B, Noutsias M, Ruppert V, Richter A, Pankuweit S (2012) Cardiomyopathies: classification, diagnosis, and treatment. Heart Failure Clin 8:53-78

7. Sosa E, Scanavacc M, d'Avila A (1996) A new technique to perform epicardial mapping in the electrophysiology laboratory. J Cardiovasc Electrophysiol 7(6):531-536

8. Sosa E, Scanavacca M, d'Avila A (1999) Different ways of approaching the normal pericardial space. Circulation 100(24): e115-e116

9. Stoll HP, Carlson K, Keefer LK, Hrabie JA, March KL (1999) Pharmacokinetics and consistency of pericardial devlivery directed to coronary arteries: direct comparison with endoluminal devlivers. Clin Cardiol 22(1 Suppl 1):110-116

10. Rupp H, Rupp O, Alter P, Jung N, Pankuweit S, Maisch B (2010) Regeneration by stem cells. Need for minimal invasive access(AttachLifter) the normal pericardial cavity. Herz 7:458-466

11. Ota T, Patronik NA, Schwarzman D, Riviere CN, Zenat MI (2008) Minimally invasive epicardial injections using a novel semiautonomous robotic device. Circulation 118(14 Suppl):115-120

12. Sagrista-Sauleda J, Permanyer-Miralda G, Candell-Riera J, Angel J, Soler-Soler J (1987) Transient cardiac constriction: an unrecognized pattern of evolution in effusive acute idiopathic pericarditis. Am J Cardiol 59:961-966

13. Oh JK, Hatle LK, Mulvagh SL, Tajik AJ (1993) Transient constrictive pericarditis: diagnosis by two-dimensional Doppler echocardiography. Mayo Clin Proc 68:1158-1164

14. Feng D, Glockner J, Kim K, Martinez M, Syed IS, Araoz P, Breen J, Espinosa RE, Sundt T, Schaff HV, Oh JK (2011) Cardiac magnetic resonance imaging pericardial late gadolinium enhancement and elevated inflammatory markers can predict the reversibility of constrictive pericarditis after antiinflammatory medical therapy: a pilot study. Circulation 124:1830-1837

15. Veress G, Kim KH, Masaki M, Espinosa RE, Oh JK (2010) Differential diagnosis of constrictive pericarditis from restrictive myocardial disease by speckle tracking echocardiography. J Am Coll Cardiol 55:10A

16. Rienmuller R, Groll R, Lipton MJ (2004) CT and MR imaging of pericardial disease. Radiol Clin North Am 42(3):587-601

17. Maksimovic R, Dill T, Seferovic PM, Ristic AD, Alter P, Simeunovic DS, Markovic Z, Bachmann GF, Maisch B (2006) Magnetic resonance imaging in pericardial diseases. Indications and diagnostic value. Herz 31(7):708-714

18. Torrance DJ (1955) Demonstration of subepicardial fat as an aid in the diagnosis of pericardial effusion or thickening. Am J Roentgenol 1955(74):850-855

19. Maisch B, Ristić AD, Karatolios K (2011) Pericardiocentesis. In: Tubaro M, Danchin N, Filippatos G, Goldstein P, Vranickx P, Zahger D (eds) The ESC textbook of intensive and acute cardiac care. Oxford University Press, Oxford, pp 246-256

20. Seferovic PM, Ristic AD, Maksimovic R, Tatic V, Ostojic M, Kanjuh V (2003) Diagnostic value of pericardial biopsy: improvement with extensive sampling enabled by pericardioscopy. Circulation 107:978-983

21. Maisch B, Drude L (1991) Pericardioscopy—a new diagnostic tool in inflammatory diseases of the pericardium. Eur Heart $\mathrm{J}$ 12(Suppl. D):2-6

22. Maisch B, Bethge C, Drude L, Hufnagel G, Herzum M, Schönian (1994) Pericardioscopy and epicardial biopsy-new diagnostic tools in pericardial and perimyocardial disease. Eur Heart $\mathrm{J}$ 15(Suppl. D):68-73

23. Maisch B, Ristic AD, Pankuweit S, Neubauer A, Moll R (2002) Neoplastic pericardial effusion: efficacy and safety of intrapericardial treatment with cisplatin. Eur Heart J 23:1625-1631 
24. Maisch B, Ristic A, Pankuweit S (2010) Evaluation and management of pericardial effusion in patients with neoplastic disease. Prog Cardiovasc Dis 53(2):157-163

25. Maisch B, Ristic AD, Pankuweit S (2002) Intrapericardial treatment of autoreactive pericardial effusion with triamcinolone: the way to avoid side effects of systemic corticosteroid therapy. Eur Heart J 23:1503-1508

26. Pankuweit S, Moll R, Baantrup U, Portig I, Hufnagel G, Maisch B (2003) Prevalence of the Parvovirus B19 genome in endomyocardial biopsy specimens. Hum Pathol 34:497-500

27. Karatolios K, Pankuweit S, Goettsch C, Hofbauer LC, Timmesfeld N, Al-Fakhri N, Maisch B, Schoppet M (2012) Osteoprotegerin (OPG) and TNF-related apoptosis-inducing ligand (TRAIL) levels in malignant and benign pericardial effusions. Clin Biochem 45(3):237-242

28. Imazio M, Bobbio M, Cecchi E, Demarie D, Demichelis B, Pomari F, Moratti M, Gaschino G, Giammaria M, Ghisio A, Belli R,
Trinchero R (2005) Colchicine in addition to conventional therapy for acute pericarditis: results of the COlchicine for acute PEricarditis (COPE) trial. Circulation 112(13):2012-2016

29. Imazio M, Bobbio M, Cecchi E et al (2005) Colchicine as firstchoice therapy for recurrent pericarditis: results of the CORE (COlchicine for REcurrent pericarditis) trial. Arch Intern Med 165:1987-1991

30. Imazio M, Brucato A, Cemin R, Ferrua S, Belli R, Maestroni S, Trinchero R, Spodick DH, Adler Y, CORP (COlchicine for Recurrent Pericarditis) Investigators (2001) Colchicine for recurrent pericarditis (CORP): a randomized trial. Ann Intern Med 155(7):409-414

31. Mayosi BM, Burgess LJ, Doubell AF (2005) Tuberculous pericarditis. Circulation 112(23):3608-3616

32. Ntsekhe M, Hakim J (2005) Impact of human immunodeficiency virus infection on cardiovascular disease in Africa. Circulation 112(23):3602-3607 